retroviruses has provided insight into many important areas of biology removed from "straight" virology. Retrovirus genomes can be inherited as Mendelian genes as well as spread between and within species by infection; they mimic the effects of mobile genetic elements by mutation and alteration of the expression of chromosomal genes; they activate, recombine with and transduce cellular oncogenes; and they induce defects in the regulation of cell growth. The book is therefore of direct interest to virologists, molecular biologists, geneticists, cell biologists, tumour biologists and clinicians.

Does the book work? The text is divided into 11 chapters which deal firstly with molecular aspects of the genome, its replication and expression, and then with the biological aspects of expression, infection and pathogenesis. The selfcontained chapter structure has resulted in considerable repetition and contributes to the overall length of the book. This is anticipated in the preface and on balance the strategy is acceptable; a little reiteration does no harm when the subject matter is so complex. The text has been researched in such formidable detail that complete novices may find some sections hard going. I imagine that undergraduates, for example, would need some guidance in the correct usage of the book. Nonetheless, the writing is generally clear and in most chapters there should be no difficulty in abstracting the information at different levels of complexity. Moreover, where doubt or ambiguity of interpretation is present, this is usually pointed out and the alternatives explained.

I particularly enjoyed the three chapters on the molecular biology of the retrovirus genome. They clearly explain the exquisite mechanisms by which the RNA genome is converted to a DNA copy in which a terminally redundant LTR sequence is created, how this is inserted into the chromosome and expressed using tactical variations on a strategy evolved to permit the synthesis of many gene products from a single genome sequence. The sections covering endogenous viruses also successfully deal with a complex subject while highlighting the evolutionary implications. Of course the pathogenicity, particularly the oncogenicity, of retroviruses is currently a centre of attention and readers will find the chapters on pathogenicity and oncogenes to be of

\section{An English Lamarck}

An English translation of Madeleine Barthélemy-Madaule's Lamarck oule Mythe du Précurseur (Editions du Seuil, 1979) has just been published by MIT Press. The original edition was reviewed in Nature 286, $187 ; 1980$

The translation (by M.H. Shank) has the title Lamarck the Mythical Precursor, and costs $\$ 17.50, £ 12.25$. especial interest. These subjects are, in the main, well treated. Just enough (and no more) information on haematopoiesis is given to understand the concepts involved in virus-induced cellular disease. The several current models for the mechanistic interpretation of transformation are fairly represented and the review of oncogenes and their products is also well-balanced and up-to-date.

The final chapter on the identification of human RNA tumour viruses explains the dangers of overinterpreting initial results and introduces the only convincing isolate to date: the lymphoma-leukaemia virus associated with certain forms of adult T-cell leukaemia. A sting in the tail of the book is provided by a compilation of restriction endonuclease maps and primary nucleotide and amino acid sequences of some well-studied viruses.

Students of retrovirology and its practitioners will be not unaware of the clashes which often arise between some dominant personalities with individual and exclusive viewpoints. Although only a small number of authors could be included in the writing of the current volume, my impression is that this has not been allowed to disturb the balance of the text and that the views expressed are reasonably representative of the field (not always the case in other volumes from the same stable).

I also sense that the timing of the book is critical: we are probably ending a phase in which details of the virus structure are the main concern, and beginning one in which the consequences of virus-cell interactions will predominate. This volume comfortably spans these areas. It is exhaustively researched and referenced, and will provide the standard reference text for the field for the foreseeable future.

Neil Wilkie is Head of the Tumour Virus Laboratory at the Beatson Institute for Cancer Research, Glasgow.

\title{
Looking at comets in Arizona
}

\section{David W. Hughes}

Comets. Edited by Laurel L. Wilkening. Pp.766. ISBN 0-8165-0769-4. (University of Arizona Press: 1982.) \$29.95.

TUCSON, Arizona was the place and March 1981 the time of the meeting of 175 scientists for the Sixty-first International Astronomical Union's Colloquium on "Comets: Gases, Ices, Grains and Plasma'. The review papers presented at that meeting provide the basis for this book.

Comets have been newsworthy for centuries and scientists are forever using the latest observational techniques and theoretical gambits to delve into their mysteries. The 1980 s are no exception. The impending return of Halley's comet in 1986 should see some visits by spacecraft; the successful operation of the International Ultraviolet Explorer has made $\mathrm{H}$ and $\mathrm{OH}$ observations in the extended coma a commonplace occurrence; the collection of cometary dust in the Earth's upper atmosphere is now routine; and the Pioneer Venus orbiter observations of the interactions of the solar wind with an atmosphere unprotected by a magnetic field gave a preview of what is to be expected in the cometary case.

"New theories and data are collected in this book as an introduction to comets for newcomers as well as a reference volume for specialists" - I quote from Laurel Wilkening's preface. Unfortunately I don't think a book can easily be both and this one certainly is not. It contains 29 papers grouped under headings such as Overview; Nucleus; Dust; Coma, Ion Tails and Solar Wind Interaction; Origin,
Evolution and Interrelations; and Basic Information and References. As with all conference proceedings the standard is mixed but there are some real gems Delsemme on the chemical composition of cometary nuclei, Kresak on statistics and observational selection, Whipple on nuclei rotations, Sekanina on splitting, Wetherill and Revelle on the relationship between comets, meteorites and fireballs, A'Hearn on spectrophotometry, Feldman on the ultraviolet, Everhart on orbital evolution, Marsden and Roemer on astrometry and basic characteristics.

Such papers lift this conference proceedings from the category of "every library should purchase it" to "every comet researcher should have it on his personal bookshelf"'. If, however, critical comparisons must be made I do think the University of Arizona did a better job on the asteroids in 1979 than they have here on the comets. (For a review of Asteroids, edited by T. Gehrels, see Nature 287, 664; 1980.)

Also, when it comes to introducing a topic to newcomers it must be ensured that certain features are not omitted. This is especially difficult for the editor of conference proceedings who is restricted not only by the ease with which scientists raise travel money, and their predilections for Arizona in March, but also by the problems of convincing the participants to write up their papers in a suitable form. There are far too many gaps in this volume to make it a good introduction for the newcomer.

David W. Hughes is a Lecturer in Astronomy and Physics at the University of Sheffield. 